\title{
Necrotizing fasciitis caused by Haemophilus influenzae type $b$ in a patient with rectal cancer treated with combined bevacizumab and chemotherapy: a case report
}

\author{
Tomotaka Ugai ${ }^{1,2^{*}}$, Masataro Norizuki ${ }^{1,3}$, Takahiro Mikawa' ${ }^{1}$ Goh Ohji ${ }^{4}$ and Makito Yaegashi ${ }^{*}$
}

\begin{abstract}
Background: Recently, necrotizing fasciitis has been reported in patients treated with bevacizumab, usually secondary to wound healing complications, gastrointestinal perforations, or fistula formation. The risk of invasive Haemophilus influenzae type b infection is significantly increased in immunocompromised hosts. However, necrotizing fasciitis due to Haemophilus influenzae type $b$ in a patient treated with combined bevacizumab and chemotherapy has not been previously reported.

Case presentation: A 59-year-old woman was admitted to the intensive care unit after sudden onset of fever, chills, and right thigh pain. She received chemotherapy with fluorouracil, irinotecan, and bevacizumab for colon cancer 10 days prior to admission. The advancing erythematous margin and her worsening clinical condition prompted us to suspect necrotizing fasciitis and consult the orthopedics department for a fascia biopsy and debridement. Surgical exploration revealed a murky dishwater-colored pus exudate from the incision site and the lack of a shiny appearance of the fascia that also suggested necrotizing fasciitis. After 2 days, the final results of the blood and exudate cultures confirmed the presence of Haemophilus influenzae type b. A diagnosis of necrotizing fasciitis due to Haemophilus influenzae type $b$ was made. The patient required recurrent surgical debridement and drainage, but she recovered from the septic shock.
\end{abstract}

Conclusions: We report a case of necrotizing fasciitis due to Haemophilus influenzae type $b$ in a patient without injury and with rectal cancer treated with combined bevacizumab and chemotherapy. Physicians should consider invasive Haemophilus influenzae type $b$ disease in the presence of necrotizing fasciitis in patients treated with this combined treatment modality.

Keywords: Necrotizing fasciitis, Haemophilus influenzae type b, Bevacizumab

\section{Background}

Bevacizumab is an antibody designed to inhibit vascular endothelial growth factor, which is involved in blood vessel formation, and used primarily in the cancer field for the treatment of colorectal cancer to prevent the formation of blood vessels that feed tumors. Recently,

\footnotetext{
*Correspondence: harukakanata58@yahoo.co.jp; myaegashi@hotmail.com ${ }^{1}$ Division of General Internal Medicine and Infectious Disease, Department of Medicine, Kameda Medical Center, Kamogawa-shi, Chiba 296-8601, Japan ${ }^{2}$ Current address; Division of Hematology, Department of Medicine, Saitama Medical Center, Jichi Medical University, Amanuma-cho, Omiya, Saitama-shi, Saitama 330-8503, Japan

Full list of author information is available at the end of the article
}

necrotizing fasciitis has been reported in patients treated with bevacizumab, including some fatal cases [1]. However, necrotizing fasciitis usually develops secondary to wound healing complications, gastrointestinal perforations, or fistula formation. Here, we report a case of necrotizing fasciitis due to Haemophilus influenzae type b (Hib) in a patient without injury and with rectal cancer treated with combined bevacizumab and chemotherapy.

\section{Case presentation}

A 59-year-old woman was admitted to the intensive care unit after sudden onset of fever, chills, and right thigh 
pain. She had undergone low anterior resection for colon cancer 2 years earlier. After local recurrence, she underwent radiation therapy (30 Gy) for spinal metastasis followed by chemotherapy, which commenced 6 months prior to admission to the intensive care unit. She received chemotherapy with fluorouracil, irinotecan, and bevacizumab 10 days prior to admission. She did not have a history of previous injury or the presence of other risk factors for necrotizing fasciitis such as a decubitus ulcer, diabetes, or liver cirrhosis. Her medications included a 5-HT3 receptor antagonist, which is an antiemetic, and a proton pump inhibitor; however, her medications did not include non-steroidal anti-inflammatory drugs, immunosuppressive drugs, or corticosteroids.

She was hypotensive, tachycardic, and tachypneic in the emergency room. The results of the physical examination were normal except for erythema in her right thigh. She had no previous injuries. Hemoglobin level was $118 \mathrm{~g} / \mathrm{L}$ with a white blood cell count of $2.1 \times 10^{9}$ cells $/ \mathrm{L}$ and a platelet count of $1.3 \times 10^{11}$ cells/L. Blood urea nitrogen was $18.9 \mathrm{mmol} / \mathrm{L}$, creatinine was $318.2 \mu \mathrm{mol} / \mathrm{L}$, and C-reactive protein was $3.8 \times 10^{6} \mu \mathrm{g} / \mathrm{L}$. The results of other blood tests and urinalysis were normal. Computed tomography of the chest, abdomen, and pelvis showed normal results. Septic shock was suspected and managed accordingly with supportive measures, such as the use of a vasopressor and supplemental oxygen. Blood cultures were collected, and empirical treatment with meropenem (1 g every 12 hours) and vancomycin ( $1 \mathrm{~g}$ daily) were initiated, adjusted to renal dysfunction. The advancing erythematous margin and her worsening clinical condition prompted us to suspect necrotizing fasciitis and consult the orthopedics department for a fascia biopsy and debridement. Surgical exploration revealed a murky dishwater-colored pus exudate from the incision site and the lack of a shiny appearance of the fascia that also suggested necrotizing fasciitis. The biopsy of the frozen section showed acute necrotizing suppurative inflammation, and extensive debridement was performed. Gram staining of the exudates from the infected site showed the presence of gram-negative rods. On the basis of all of this information, we formally diagnosed the patient with necrotizing fasciitis. On the following day, gram-negative rods were also isolated from the blood cultures. After 2 days, the final results of the blood and exudate cultures confirmed the presence of $\beta$-lactamase-negative Hib that was susceptible to ampicillin, cefotaxime, ciprofloxacin, and imipenem. The histopathological findings of the debrided tissue were compatible with a diagnosis of necrotizing fasciitis due to Hib.

The patient required recurrent surgical debridement and drainage, but she recovered from the septic shock. The treatment was changed to ampicillin, and she received intravenous ampicillin for a total of 51 days because of residual intermuscular abscesses. She recovered completely and was discharged from the hospital 65 days after admission. Necrotizing fasciitis did not recur; however, she died of metastatic colorectal cancer 6 months after discharge.

\section{Discussion}

Necrotizing fasciitis is a rare but life-threatening infection of the soft tissue that is characterized by rapidly spreading necrosis of the superficial fascia and subcutaneous tissue. Immunocompromised and diabetic patients are at a higher risk of developing necrotizing fasciitis [2]. Although bevacizumab suppresses the immune system [3], chemotherapy administered concurrently with bevacizumab is more likely to be responsible for immune suppression. This combined treatment also contributes to impaired wound healing. Furthermore, one of the pathophysiologic mechanisms of necrotizing fasciitis is subcutaneous artery thrombosis and tissue ischemia, to which bevacizumab can contribute [4]. Therefore, the combined treatment modality of bevacizumab and chemotherapy is likely to place patients at an increased risk of developing necrotizing fasciitis. Despite the fact that necrotizing fasciitis is a rare complication, affecting only 1 in 5000 bevacizumab users, physicians should bear in mind the risk of necrotizing fasciitis when prescribing bevacizumab.

In a comprehensive safety review conducted by the company Roche, 52 case reports of serious necrotizing fasciitis were identified that occurred between November 1997 and September 2012 in patients treated with bevacizumab for cancer [5]. The majority of the patients described in case reports have had gastrointestinal perforations, fistula formation, or wound healing complications preceding the development of necrotizing fasciitis [6-8]. However, in the present case, the patient had no previous injury or risk factor for necrotizing fasciitis such as a decubitus ulcer, diabetes, or liver cirrhosis; her recent chemotherapy was her primary risk for necrotizing fasciitis. These findings suggest a possible association between necrotizing fasciitis and the recent combined treatment of chemotherapy and bevacizumab in the absence of a previous injury.

It is of particular interest that Hib caused the necrotizing fasciitis in the present case. Prior to routine Hib vaccination, Hib was a well-known cause of invasive diseases, such as meningitis and pneumonia with bacteremia, in children younger than 2 years. However, the incidence of invasive $H$. influenzae disease, especially among persons aged over 65 years, and invasive non-typable $H$. influenzae disease has increased from 1996 to 2004 [9]. The risk of invasive Hib infection in adults is significantly increased by multiple myeloma and chronic renal failure [10]. Other factors conferring increased risk for $H$. influenzae infection include underlying immunocompromising conditions 
such as complement deficiency, hypogammaglobulinemia, sickle cell anemia, functional asplenia, malignancy, and human immunodeficiency virus infection [11,12]. Although the association between bevacizumab and invasive Hib infection remains unclear, we caution physicians to consider invasive Hib disease in patients treated with the combination of bevacizumab and chemotherapy.

A review of the current literature resulted in only 4 reported cases of necrotizing fasciitis caused by Hib. The first reported case occurred in a 13-month-old infant [13], while the second case occurred in an 81-year-old man with diabetes mellitus [14]. A 64-year-old woman who developed necrotizing fasciitis of her chest wall secondary to the epiglottitis represented the third case [15]. In the fourth case, a 44-year-old man developed necrotizing fasciitis of the right lower extremity after intramuscular injections of paracetamol in his right buttock [16]. Including our case, all of the patients with necrotizing fasciitis due to Hib made a full recovery. Hib merits additional consideration when deciding on appropriate, empiric antimicrobial therapy as an adjunct to surgical intervention for necrotizing fasciitis, especially in immunocompromised patients treated with combined bevacizumab and chemotherapy.

\section{Conclusions}

We report a case of necrotizing fasciitis due to Hib in a patient without injury and with rectal cancer treated with combined bevacizumab and chemotherapy. Physicians should consider invasive Hib disease in the presence of necrotizing fasciitis in patients treated with this combined treatment modality.

\section{Consent}

Written informed consent was obtained from the patient for publication of this case report. A copy of the written consent is available for review by the Editor-in-Chief of this journal.

\section{Abbreviations}

Hib: Haemophilus influenzae type b.

\section{Competing interests}

The authors declare that they have no competing interests. All authors have submitted the ICMJE Form for Disclosure of Potential Conflicts of Interest. Conflicts that the editors consider relevant to the content of the manuscript have been disclosed.

\section{Authors' contributions}

All authors were involved in the clinical care of the patient and acquisition and interpretation of the data. TU drafted the manuscript. All authors read and approved the final manuscript.

\section{Author details}

'Division of General Internal Medicine and Infectious Disease, Department of Medicine, Kameda Medical Center, Kamogawa-shi, Chiba 296-8601, Japan. ${ }^{2}$ Current address; Division of Hematology, Department of Medicine, Saitama Medical Center, Jichi Medical University, Amanuma-cho, Omiya, Saitama-shi, Saitama 330-8503, Japan. ${ }^{3}$ Current address; Department of Infectious Disease,
Jichi Medical University, Shimotsuke, Tochigi 329-0498, Japan. ${ }^{4}$ Division of Infectious Disease, Department of Microbiology and Infectious Diseases, Kobe University Graduate School of Medicine, 7-5-2 Kusunoki-cho, Chuo-ku, Kobe, Hyogo 650-0017, Japan.

Received: 14 February 2014 Accepted: 9 April 2014

Published: 12 April 2014

\section{References}

1. Shamloo BK, Chhabra P, Freedman AN, Potosky A, Malin J, Weiss Smith S: Novel adverse events of bevacizumab in the US FDA adverse event reporting system database: a disproportionality analysis. Drug Saf 2012, 35(6):507-518

2. Stumvoll M, Fritsche A: Necrotizing fasciitis caused by unencapsulated Haemophilus influenzae. Clin Infect Dis 1997, 25(2):327.

3. Correale P, Remondo C, Carbone SF, Ricci V, Migali C, Martellucci I, Licchetta A, Addeo R, Volterrani L, Gotti G, Rotundo MS, Tassone P, Sperlongano P, Abbruzzese A, Caraglia M, Tagliaferri P, Francini G: Dose/dense metronomic chemotherapy with fractioned cisplatin and oral daily etoposide enhances the anti-angiogenic effects of bevacizumab and has strong antitumor activity in advanced non-small-cell-lung cancer patients. Cancer Biol Ther 2010, 9(9):685-693.

4. Sarani B, Strong M, Pascual J, Schwab CW: Necrotizing fasciitis: current concepts and review of the literature. J Am Coll Surg 2009, 208(2):279-288.

5. Wong J: Bevacizumab is linked to cases of necrotising fasciitis. Lancet Oncol 2013, 14(7):e259.

6. Shimada A, Nakamura T, Ishii M, Chiba N, Ishikawa S, Arisawa Y, Hashimoto M: A case of necrotizing fasciitis developed in a patient with recurrent rectal cancer treated with chemotherapy. Gan To Kagaku Ryoho 2013, 40(5):663-665.

7. Gamboa EO, Rehmus EH, Haller N: Fournier's gangrene as a possible side effect of bevacizumab therapy for resected colorectal cancer. Clin Colorectal Cancer 2010, 9(1):55-58.

8. Sendur MA, Aksoy S, Ozdemir NY, Zengin N: Necrotizing fasciitis secondary to bevacizumab treatment for metastatic rectal adenocarcinoma. Indian J Pharmacol 2014, 46(1):125-126.

9. Dworkin MS, Park L, Borchardt SM: The changing epidemiology of invasive Haemophilus influenzae disease, especially in persons $>$ or $=65$ years old. Clin Infect Dis 2007, 44(6):810-816.

10. Nix EB, Hawdon N, Gravelle S, Biman B, Brigden M, Malik S, McCready W, Ferroni G, Ulanova M: Risk of invasive Haemophilus influenzae type $b$ (Hib) disease in adults with secondary immunodeficiency in the post-Hib vaccine era. Clin Vaccine Immunol 2012, 19(5):766-771.

11. Makela PH, Takala AK, Peltola H, Eskola J: Epidemiology of invasive Haemophilus influenzae type b disease. J Infect Dis 1992, 165(Suppl 1):S2-S6.

12. Cordero E, Pachon J, Rivero A, Giron JA, Gomez-Mateos J, Merino MD, Torres-Tortosa M, Gonzalez-Serrano M, Aliaga L, Collado A, Hernández-Quero J, Barrera A, Nuño E: Haemophilus influenzae pneumonia in human immunodeficiency virus-infected patients. The Grupo Andaluz para el Estudio de las Enfermedades Infecciosas. Clin Infect Dis 2000, 30(3):461-465.

13. Collette CJ, Southerland D, Corrall CJ: Necrotizing fasciitis associated with Haemophilus influenzae type b. Am J Dis Child 1987, 141(11):1146-1148.

14. Saito T, Matsunaga H, Matsumura Y, Segawa H, Takakura S, Nagao M, linuma $Y$, Miyachi Y, Ichiyama S: Necrotizing fasciitis caused by Haemophilus influenzae type b in an elderly patient. J Clin Microbiol 2009, 47(3):852-854.

15. Chalmers C: Necrotising fasciitis complicating Haemophilus influenzae type b epiglottitis in an adult. J Laryngol Otol 2010, 124(7):807-809.

16. Lee EY, Ip WY: Necrotizing fasciitis of the extremity caused by Haemophilus influenzae serotype $b$ in a healthy adult. Clin Orthop Relat Res 2010, 468(5):1436-1439.

doi:10.1186/1471-2334-14-198

Cite this article as: Ugai et al: Necrotizing fasciitis caused by

Haemophilus influenzae type $\mathrm{b}$ in a patient with rectal cancer treated with combined bevacizumab and chemotherapy: a case report. BMC Infectious Diseases 2014 14:198. 